\title{
Variabilité intraspécifique chez le pin maritime (Pinus pinaster Ait) dans le sud-est de la France. I. Variabilité des populations autochtones et des populations de l'ensemble de l'aire de l'espèce*
}

\author{
A Harfouche 1, P Baradat ${ }^{2}$, CE Durel ${ }^{1}$ \\ avec la collaboration technique de $\mathrm{J} \mathrm{Pommery}^{3}$
}

1 INRA, laboratoire de génétique et amélioration des arbres forestiers, BP 45, Gazinet, 33610 Cestas;

2 CIRAD, UR de biométrie, 2477, avenue du Val-de-Montferrand, BP 5035, 34032 Montpellier;

3 INRA, domaine expérimental du Ruscas, 82237 Bormes-les-Mimosas, France

(Reçu le 25 avril 1994 ; accepté le 2 janvier 1995)

\begin{abstract}
Résumé - Une plantation comparative de 25 provenances de pin maritime implantée en 1982 dans le sud-est de la France (massif des Maures) a été étudiée en 1988, 1989 et 1993. À l'échelle régionale, il existe une importante variabilité pour les caractères de vigueur (hauteur et diamètre) et pour la sensibilité à la cochenille Matsucoccus feytaudi mais une faible différenciation pour les caractères de forme (écart à la verticalité, coefficient de forme, angle d'insertion et grosseur des branches). Les analyses mettent, en revanche, en évidence une grande variabilité pour l'ensemble des caractères étudiés à l'échelle de l'aire de l'espèce. Les provenances atlantiques sont les plus vigoureuses et parmi les moins sensibles au ravageur. Les provenances corses sont hétérogènes pour les caractères de forme et présentent une grande sensibilité au ravageur. La provenance marocaine de Tamjout est complètement résistante à la cochenille. Les provenances du sud-est sont moyennes pour tous les caractères de forme et de vigueur mais très sensibles à $M$ feytaudi. Ces résultats posent la question de l'intérêt des populations locales pour le développement sylvicole et la mise en œuvre d'un programme d'amélioration génétique pour le sud-est de la France. L'alternative d'une hybridation interraciale comme méthode d'amélioration de l'espèce dans la région est discutée.
\end{abstract}

Pinus pinaster Ait / variabilité intraspécifique / sensibilité à Matsucoccus feytaudi

\footnotetext{
* Correspondance et tirés à part : A Kremer, INRA, laboratoire de génétique et amélioration des arbres forestiers, BP 45, Gazinet, 33610 Cestas.
} 
Summary - Intraspecific variability in maritime pine (Pinus pinaster Ait) in the south-east of France. I. Variability in autochthonous populations and in the whole range of the species. $A$ provenance trial of maritime pine comprising 25 provenances (table I) and located in the south-east of France was studied at 8,9 and 13 years of age. The results show a high variability for growth and sensibility to the coccid Matsucoccus feytaudi at the regional level, but no differentiation for form traits (see table III, analysis 1). At the species range level, however, significant provenance differences appear for all characters (see table III, analyses 2 and 3). Atlantic provenances are the most vigorous and among the most resistant to the coccid (see figs 1 and 2). Corsican provenances show an intraracial variation for form and a great sensibility to the insect (see figs 1 and 2). The Moroccan origin appears to be completely resistant to the insect, while the south-eastern populations are intermediate for vigor and form but quite sensitive to the pest (see figs 1 and 2). These results raise the problem of the use of the local populations as reproductive material in the south-east. In this respect, interracial hybridization may be an alternative method for genetic improvement of maritime pine.

\section{Pinus pinaster Ait / intraspecific variability / sensibility to Matcucoccus feytaudi}

\section{INTRODUCTION}

Les glaciations en Europe ont fortement marqué la dynamique des populations de pin maritime dont l'aire de dispersion se trouva réduite à une succession d'îlots plus ou moins étendus dans les régions sud-atlantique et méditerranéenne. La recolonisation de l'espace, à l'issue de ces périodes, se serait opérée à partir d'un groupe restreint de peuplements confinés à quelques zones refuges dont le sud de l'Espagne (Baradat et Marpeau-Bezard, 1988).

En Afrique du Nord, la distribution spatiale des populations naturelles présente encore plus de discontinuités.

Bien que des échanges de matériel génétique aient pu survenir entre populations de l'ensemble de l'aire (transfert de graines à certaines époques de l'histoire), l'isolement, ajouté à une taille souvent réduite, a probablement accentué leur divergence génétique.

Les études récentes de Baradat et Marpeau-Bezard (1988) mettent en évidence une différenciation génétique assez marquée pour des terpènes, ce qui a conduit ces auteurs à distinguer globalement 3 grands groupes géographiques: les groupes Atlantique, Périméditerranéen et Maghrébin. Ces groupes principaux se scin- dent à leur tour en races géographiques traduisant une diversité intragroupe assez importante pour ce type de marqueurs génétiques.

D'une manière générale, tous les auteurs qui ont eu à étudier des populations de pin maritime (Bouvarel, 1960 ; Hopkins, 1960 ; Sweet et Thulin, 1962 ; Illy, 1966 ; Resch, 1974 ; Kremer et Roussel, 1986 ; Baradat et Marpeau-Bezard, 1988 ; Petit, 1988 ; Bahrman et al, 1992) s'accordent à y voir une importante variabilité. Notre étude est une contribution supplémentaire à l'étude de cette variabilité. Elle s'inscrit, et c'est là son originalité par rapport aux études précédentes, dans un contexte particulier qui est celui du sud-est de la France, où le pin maritime a été décimé par la cochenille Matsucoccus feytaudi Duc (Homoptera: Margarodidae). L'évolution du dépérissement, les perspectives de régulation naturelle des infestations ainsi que les problèmes posés par la lutte contre le ravageur ont été clairement définis dans l'article de Schvester (1981). II reste au plan génétique à approcher des questions telles la variabilité et le déterminisme génétiques de la sensibilité et à explorer l'éventualité d'une existence de gènes de résistance à l'insecte dans les populations naturelles de pin maritime. C'est là l'un des objectifs premiers de notre étude. 
La disponibilité de provenances originaires de l'ensemble de l'aire naturelle et un échantillonnage particulièrement dense des populations locales permettent d'effectuer une analyse de la variabilité intraspécifique de l'espèce sous divers aspects :

- i) À l'échelon régional, afin d'examiner si les populations autochtones présentent ou non un degré de différenciation significatif qui en ferait des entités significativement distinctes les unes des autres.

- ii) À l'échelle de l'aire de l'espèce, par l'étude d'un échantillon de races et provenances couvrant la majeure partie de cette aire.

- iii) Et, enfin, aux 2 échelles, en comparant les variabilités régionale et globale et les amplitudes de variation sous-jacentes.

Les résultats permettront notamment de juger la valeur des populations locales par rapport à des provenances «exotiques" et par suite l'intérêt d'impliquer ou non certaines d'entre elles dans un éventuel programme d'amélioration génétique de l'espèce pour la région.

Le présent article est le premier d'une série sur les thèmes de la variabilité intraspécifique et de l'hybridation interraciale chez le pin maritime dans les 2 régions du sud-est et du sud-ouest de la France. Les recherches s'appuient sur l'analyse d'une plantation comparative bistationnelle (installée dans le massif des Maures et dans les Landes) comportant à la fois des provenances de l'ensemble de l'aire du pin maritime et leurs hybrides réalisés par croisements contrôlés.

\section{MATÉRIELS ET MÉTHODES}

\section{Échantillonnage de races et de provenances}

Vingt-cinq provenances appartenant à diverses races représentant l'ensemble des 3 groupes décrits par Baradat et Marpeau-Bezard (1988) sont impliquées dans l'expérience (tableau I). Trois remarques peuvent être faites :

-i) Sur-échantillonnage des populations autochtones du sud-est de la France avec 12 provenances au total.

-ii) Les provenances du Gard (Portes: POR(a)) et des Corbières (Lesignan $1:$ BOU(a) et $2:$ ROC(a) et Fonfroide : FON(a)) seraient, d'après leur profil terpénique, d'origine artificielle (signalée par (a)). Les premières descendraient de populations du sud du Portugal alors que les secondes se rattacheraient au massif aquitain ; toutes appartiendraient donc au groupe atlantique (Baradat et Marpeau-Bezard, 1988) ;

- iii) Porto-Vecchio, Vivario, Navaleno et Estérel ont été reconstituées par polycross à partir de 5 arbres pollinisés par un mélange de pollen de 10 arbres de la même provenance en plantation comparative dans les Landes. Toutes les autres provenances sont issues de lots de graines récoltés dans l'aire naturelle. Cette récolte s'est effectuée sur une trentaine d'arbres "tout-venants" ; c'est-à-dire n'ayant fait l'objet d'aucune forme de sélection, mais toujours suffisamment éloignés les uns des autres de manière à minimiser les probabilités d'apparentement ou d'interpollinisation. Les graines ainsi récoltées ont été réunies en un seul lot.

\section{Dispositif expérimental}

La plantation est située en forêt domaniale des Maures (La Mole) dans le département du Var. II s'agit d'un versant sud développant un sol brun de profondeur variable. Le milieu serait limitant pour le pin maritime notamment en ce qui concerne la longueur de la période de sécheresse estivale. Le dispositif est en blocs incomplets complètement randomisés non équilibré ( 3 à 18 répétitions). Chaque bloc est divisé en 20 parcelles unitaires de 4 individus de la même famille disposés linéairement.

Les semis en godet ont été effectués en pépinière du Ruscas (massif des Maures) en juin 1981 et plantés en janvier 1982. Les plants ont été installés à $1,2 \mathrm{~m}$ les uns des autres sur des lignes de labour espacées de $4 \mathrm{~m}$. À l'exclusion de tout autre traitement sylvicole, des débroussaillages ont été réalisés à l'occasion des campagnes de mesures. 
Tableau I. Situation et caractéristiques écologiques des zones de provenances.

\begin{tabular}{|c|c|c|c|c|c|c|c|}
\hline $\begin{array}{l}\text { No } \\
\text { code }\end{array}$ & $\begin{array}{l}\text { Groupe } \\
\text { géographique }\end{array}$ & Race & $\begin{array}{l}\text { Provenance } \\
\text { (abréviation) }\end{array}$ & $\begin{array}{l}\text { Zone géographique } \\
\text { et écologie } \\
\text { de la station }\end{array}$ & Latitude & Longitude & $\begin{array}{c}\text { Altitude } \\
(m)\end{array}$ \\
\hline & & & & & -. & $-\cdots$ & - \\
\hline $2^{(2)}$ & Atlantique & Aquitaine & $\begin{array}{l}\text { Landes-nord } \\
\text { (LAN) }\end{array}$ & $\begin{array}{l}\text { Médoc. } \\
\text { Dune et lande }\end{array}$ & $45^{\circ} 08^{\prime} \mathrm{N}$ & $1^{\circ} 00^{\prime} \mathrm{W}$ & 40 \\
\hline $4^{(2)}$ & Périméditerranéen & Corse & $\begin{array}{l}\text { Porto-Vecchio } \\
\text { (POV) }\end{array}$ & $\begin{array}{l}\text { Corse du Sud. } \\
\text { Sol brun superficiel, } \\
\text { roche-mère siliceuse }\end{array}$ & $41^{\circ} 39^{\prime} \mathrm{N}$ & $9^{\circ} 14^{\prime} \mathrm{E}$ & 150 \\
\hline $5(2)$ & Périméditerranéen & Corse & $\begin{array}{l}\text { Vivario } \\
\text { (VIV) }\end{array}$ & $\begin{array}{l}\text { Corse du Nord. } \\
\text { Sol brun superficiel, } \\
\text { roche-mère siliceuse }\end{array}$ & $42^{\circ} 09^{\prime} \mathrm{N}$ & $9^{\circ} 08^{\prime} E$ & 600 \\
\hline $7^{(2)}$ & Périméditerranéen & Andalousie & $\begin{array}{l}\text { Cazorla } \\
\text { (CAZ) }\end{array}$ & $\begin{array}{l}\text { Sierra andalouse. } \\
\text { Sol décarbonaté, } \\
769 \mathrm{~mm} / \mathrm{an}\end{array}$ & $37^{\circ} 32^{\prime} \mathrm{N}$ & $3^{\circ} 30^{\prime} \mathrm{W}$ & 120 \\
\hline $8^{(2)}$ & Atlantique & Vieille Castille & $\begin{array}{l}\text { Navaleno } \\
\text { (NAV) }\end{array}$ & $\begin{array}{l}\text { Sud de la Vieille } \\
\text { Castille. } \\
424 \mathrm{~mm} / \mathrm{an}\end{array}$ & $41^{\circ} 24^{\prime} \mathrm{N}$ & $4^{\circ} 20^{\prime} \mathrm{W}$ & 870 \\
\hline $9(1,2)$ & Périméditerranéen & $\begin{array}{l}\text { France } \\
\text { du sud-est }\end{array}$ & $\begin{array}{l}\text { Esterel } \\
\text { (EST) }\end{array}$ & $\begin{array}{l}\text { Massif de l'Esterel. } \\
\text { Sol brun superficiel } \\
\text { sur grès }\end{array}$ & $43^{\circ} 13^{\prime} \mathrm{N}$ & $6^{\circ} 50^{\prime} \mathrm{E}$ & 410 \\
\hline $13^{(2)}$ & Maghrébin & $\begin{array}{l}\text { Atlas } \\
\text { marocain }\end{array}$ & $\begin{array}{l}\text { Tamjout } \\
\text { (TAM) }\end{array}$ & $\begin{array}{l}\text { Moyen Atlas (Taza). } \\
\text { Sol brun superficiel } \\
\text { sur grès calcaire, } \\
650 \mathrm{~mm} / \mathrm{an}\end{array}$ & $33^{\circ} 52^{\prime} \mathrm{N}$ & $4^{\circ} 02^{\prime} \mathrm{W}$ & 1600 \\
\hline $15(2)$ & Atlantique & $\begin{array}{l}\text { Portugal } \\
\text { «type Leiria» }\end{array}$ & $\begin{array}{l}\text { Leiria } \\
(L E \mid)\end{array}$ & $\begin{array}{l}\text { Nord Portugal. } \\
\text { Sur vieille dune, } \\
764 \mathrm{~mm} / \mathrm{an}\end{array}$ & $39^{\circ} 49^{\prime} \mathrm{N}$ & $8^{\circ} 51^{\prime} \mathrm{W}$ & 50 \\
\hline $16^{(2)}$ & Maghrébin & Tunisie & $\begin{array}{l}\text { Tabarka } \\
\text { (TAB) }\end{array}$ & $\begin{array}{l}\text { Littoral algéro-tunisien. } \\
\text { Sol brun superficiel } \\
\text { sur grès, } 1044 \mathrm{~mm} / \mathrm{an}\end{array}$ & $3657 ' N$ & $8^{\circ} 46^{\prime} E$ & 200 \\
\hline $17^{(1,2)}$ & Périméditerranéen & $\begin{array}{l}\text { France } \\
\text { du sud-est }\end{array}$ & $\begin{array}{l}\text { La Mole } \\
\text { (MOL) }\end{array}$ & $\begin{array}{l}\text { Massif des Maures } \\
\text { (Var). } \\
\text { Sol brun sur schistes }\end{array}$ & $43^{\circ} 10^{\prime} \mathrm{N}$ & $6^{\circ} 33^{\prime} \mathrm{E}$ & 250 \\
\hline $18(1)$ & Périméditerranéen & $\begin{array}{l}\text { France } \\
\text { du sud-est }\end{array}$ & $\begin{array}{l}\text { Lambert } \\
\text { (LAM) }\end{array}$ & $\begin{array}{l}\text { Massif des Maures } \\
\text { (Var). } \\
\text { Argile sur schistes }\end{array}$ & $43^{\circ} 15^{\prime} \mathrm{N}$ & $6^{\circ} 24^{\prime} \mathrm{E}$ & 550 \\
\hline $19(1)$ & Périméditerranéen & $\begin{array}{l}\text { France } \\
\text { du sud-est }\end{array}$ & $\begin{array}{l}\text { Le Treps } \\
\text { (TRE) }\end{array}$ & $\begin{array}{l}\text { Massif des Maures } \\
\text { (Var). } \\
\text { Argile sur schistes }\end{array}$ & $43^{\circ} 17^{\prime} \mathrm{N}$ & $6^{\circ} 20^{\prime} E$ & 450 \\
\hline
\end{tabular}


Tableau I. Suite

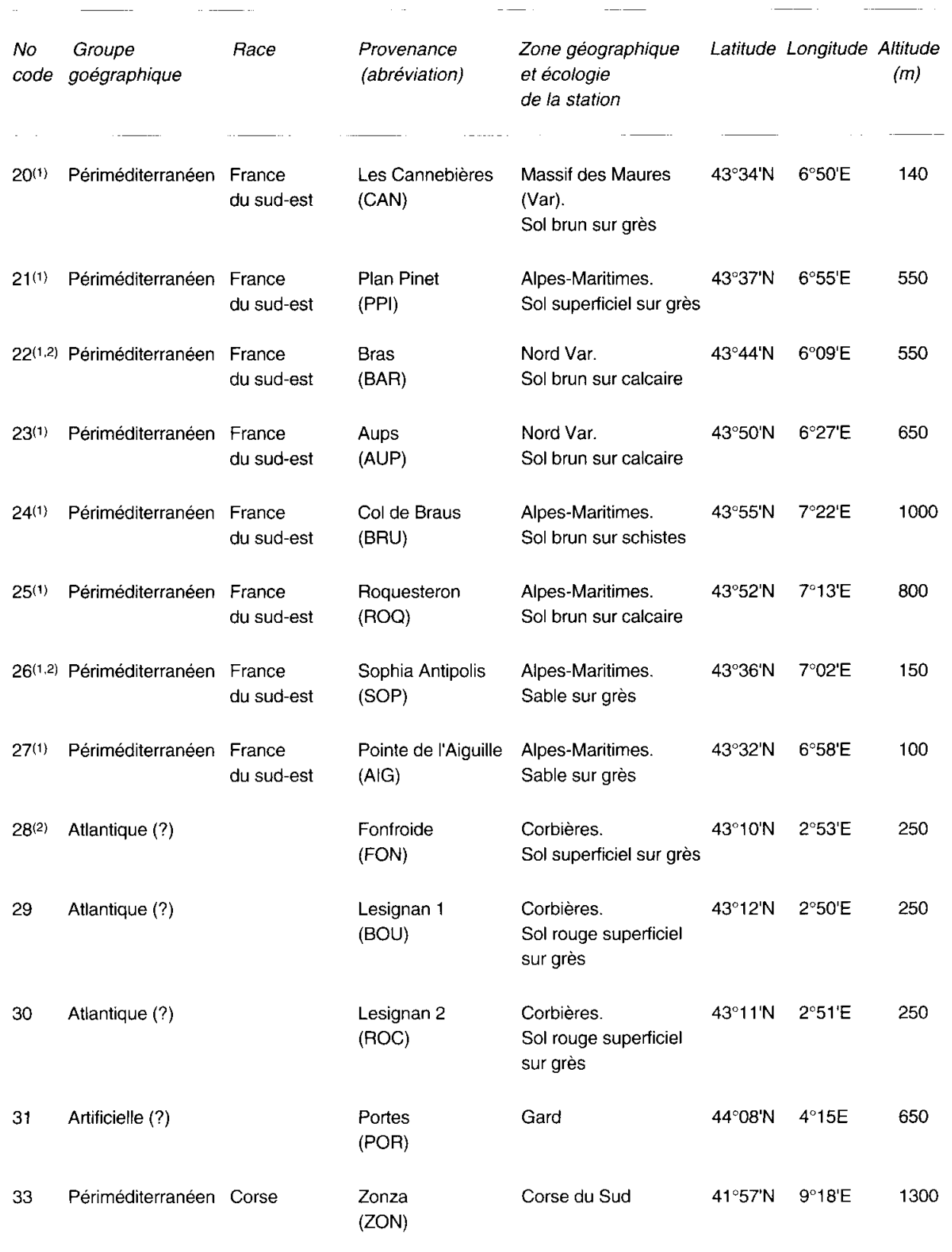

Provenances impliquées (1) dans l'analyse 1, (2) dans l'analyse 2, toutes l'étant dans l'analyse 3. 


\section{Mesures et caractères observés}

À la suite d'une forte mortalité, la provenance Tabarka (Tunisie) n'intervient dans les analyses que pour les caractères mesurés ou observés en 1988 et 1989 ainsi que pour les taux de survie. Trois campagnes de mesures ont été réalisées, en 1988,1989 et 1993, donc sur des arbres de 8, 9 et 13 ans. Les observations et mesures effectuées sont rapportées dans le tableau II.

Pour la sensibilité à $M$ feytaudi des notations de degré d'attaque ont été réalisées selon une échelle à 5 notes. D'autre part, la fréquence des individus complètement sains (note $=1$ ) a été calculée.

Tableau II. Observations et mesures.

Campagne 1988

Campagne 1989

Hauteur totale (H88)

Hauteur totale (H89)

Écart angulaire

Taux de survie 1989

à la verticalité (EV88)

(SUR89)
Taux de survie 1988

(SUR88)
Campagne 1993

Hauteur totale ( $\mathrm{H} 93)$

Diamètre à $1,30 \mathrm{~m}$ du sol (D1,30)

Diamètre à mi-hauteur $(\mathrm{DO}, 5)$

Coefficient de forme $: \uparrow$

Angle d'insertion des verticilles inférieurs (AGVI) Angle d'insertion des verticilles supérieurs (AGVS)

Note $=1:$ angle $>75^{\circ}$

2 : angle 75 à $60^{\circ}$

3 : angle 60 à $45^{\circ}$

4 : angle 45 à $30^{\circ}$

5 : angle $<30^{\circ}$

Grosseur des verticilles inférieurs (GRVI)

Grosseur des verticilles supérieurs (GRVS)

Note $=1:$ branches très fines

2 : branches fines

3 : branches moyennes

4 : branches grosses

5 : branches très grosses

Sensibilitè à Matsucoccus feytaudi (MATCOC)

Note $=1:$ individu sain

2 : individu présentant quelques coulées de résine

3 : individu présentant des coulées massives de résine

4 : individu présentant, en plus des coulées massives de résine, un jaunissement des aiguilles sur plus du tiers du houppier (individu dépérissant) 5 : individu complètement dépéri (mort)

Taux de survie 1993

(SUR93) 


\section{Traitement analytique des données}

\section{Étude exploratoire des distributions de fréquences observées}

Un examen des distributions observées et des paramètres statistiques a été effectuée en vue de définir, si possible, les lois de distribution des caractères étudiés. Ainsi, les caractères observés par échelle de notation de 1 à 5 (AGVI, AGVS, GRVI, GRVS, MATCOC) et EV88 suivent approximativement une distribution de Poisson. Ces caractères ont été transformés par la fonction $y=\sqrt{ } x$ de façon à améliorer la normalité des distributions et stabiliser la variance résiduelle (Snedecor et Cochran, 1957 ; Dagnelie, 1973 ; Lellouch et Lazar, 1974). Dans le même but, les caractères dénombrés en pourcentage (SUR89 et SUR93) ont été transformés par la fonction :

$$
y=\arcsin \sqrt{p / 100}
$$

\section{Variabilité monocaractère entre provenances}

Cette étude est conduite de 3 manières complémentaires pour répondre aux objectifs définis en introduction: i) Analyse 1 : Variabilité à l'échelle régionale sur la base des 12 provenances autochtones ; ii) Analyse 2 : Variabilité sur l'ensemble de l'aire à partir d'un échantillon de 13 provenances ; iii) Analyse 3 : Étude globale de la variabilité intraspécifique et essai de classification de l'ensemble des provenances représentées dans l'essai.

Le tableau 1 indique les provenances impliquées dans les différentes analyses. Les observations individuelles ont été ajustées à l'effet bloc selon un modèle d'analyse de variance à 2 classifications (bloc : $b_{j}$; population (ce terme englobe à la fois les provenances récoltées in situ et les descendances issues des croisements contrôlés) : $p_{i}$ ) s'écrivant comme suit :

$$
Y_{i j l}=\mu+p_{i}+b_{j}+(p b)_{i j}+e_{i j t}
$$

où $Y_{i j l}$ est la valeur de l'individu / de la population $i$ dans le bloc $j$, et $(p b)_{i j}$ un terme «interaction" intégrant un effet parcelle et l'effet d'interaction proprement dit lorsque celle-ci existe.

Les données ainsi ajustées ont été traitées par un modèle d'analyse de variance multivariable non orthogonale à un facteur (provenance, $p_{k}$ ) de la façon suivante :

$$
Y_{k l}=Y_{i j l}-\hat{b}_{j}=\mu+p_{k}+e_{k l}^{\prime}
$$

où $Y_{k l}$ est la valeur estimée de l'individu / dans la provenance $k$ après ajustement à l'effet bloc considéré comme aléatoire, $\mu$ est la moyenne générale ; $p_{k}$, l'effet de la provenance $k ; Y_{i j l}$ a la même signification que précédemment; $\hat{b}_{j}$ est l'estimation aux moindres carrés de l'effet bloc soustrait de la valeur individuelle mesurée et telle que $\sum_{i} n_{. j} . b_{j}=0, e_{k l}^{\prime}$ est le terme d'erreur intraprovenance qui intègre l'erreur vraie et l'interaction bloc $\mathrm{x}$ population telle que définie précédemment. Les $e_{k j}^{\prime}$ sont des variables aléatoires normales indépendantes, d'espérance nulle.

Ce modèle est appliqué pour les 3 types d'analyse ci-dessus. En outre, une comparaison multiple de moyennes a été réalisée selon la méthode de Newman-Keuls $(\alpha=0,05)$. Cette méthode n'étant rigoureuse que pour comparer des traitements équirépétés (erreur standard constante), on s'est rapproché des conditions normales d'utilisation en définissant comme erreur standard commune à tous les traitements: $E S=\sqrt{ } C M E / n_{h}$ où $n_{h}$ est la moyenne harmonique des effectifs par provenance et CME le carré moyen de l'erreur. Pour les analyses de variance, les hypothèses sont, $a$ priori, supposées vraies; la taille des échantillons étant trop faible pour tester leur validité.

\section{Liaison entre caractères}

Les liaisons entre caractères ont été étudiées à partir des corrélations entre estimations des effets provenance. En outre, une régression multiple descendante de la note moyenne de sensibilité à Matsucoccus feytaudi sur les variables géographiques : latitude, longitude et altitude a été effectuée en vue de mettre en évidence d'éventuelles liaisons et de compléter l'étude de la structuration géographique de l'espèce pour ce caractère effectuée sur la base du taux d'individus sains.

\section{Discrimination multicaractère des races et provenances}

Nous avons effectué une analyse factorielle discriminante afin d'étudier la structuration multicaractère des populations à l'échelle de l'aire de l'espèce. Au préalable, nous avons testé I'homogénéité des matrices de variance-covariance intrapopulation à l'aide d'un test de Kullback $\left(\chi^{2}=\right.$ 1873,6 à 1785 ddl, non significatif au seuil de risque de $5 \%$ ). 


\section{RÉSULTATS}

\section{Variabilité monocaractère entre provenances}

Les résultats de l'analyse de variance à un facteur (provenance) ainsi que les valeurs du rapport $\sigma_{p}^{2} / \sigma_{f}^{2}$ (où $\sigma_{p}^{2}$ et $\sigma_{f}^{2}$ sont, respectivement, la variance de l'effet provenance et la variance totale) pour chacune des 3 analyses sont donnés dans le tableau III. On y relèvera la forte variabilité interprovenance, à quel que niveau que ce soit de l'aire, pour les caractères de vigueur (hauteurs et diamètres), la sensibilité à Mat-

Tableau III. Analyse de variance à un facteur (provenance) : résultats pour les 3 types d'analyse.

\begin{tabular}{|c|c|c|c|c|c|c|c|}
\hline & H88 & $H 89$ & H93 & $D 1,3(93)$ & $D 0,5(93)$ & $f(93)$ & EV88 \\
\hline & -.- & & & & $\cdots$ & & .... \\
\hline \multicolumn{8}{|l|}{ Analyse 1} \\
\hline $\mathrm{F}$ & 3,81 & 4,61 & 4,47 & 2,57 & 2,51 & 1,31 & 1,05 \\
\hline$p(\%)$ & 0,004 & 0,000 & 0,000 & 0,369 & 0,466 & 21,25 & 39,97 \\
\hline $\mathrm{D}=\sigma_{p}^{2} / \sigma_{f}^{2}$ & 0,073 & 0,091 & 0,088 & 0,042 & 0,040 & 0,009 & 0,001 \\
\hline \multicolumn{8}{|l|}{ Analyse 2} \\
\hline F & 19,40 & 23,20 & 25,60 & 7,75 & 4,40 & 2,54 & 12,98 \\
\hline$p(\%)$ & 0,000 & 0,000 & 0,000 & 0,000 & 0,000 & 0,400 & 0,000 \\
\hline $\mathrm{D}=\sigma_{p}^{2} / \sigma_{f}^{2}$ & 0,305 & 0,346 & 0,370 & 0,139 & 0,075 & 0,035 & 0,222 \\
\hline \multicolumn{8}{|l|}{ Analyse 3} \\
\hline $\mathrm{F}$ & 17,10 & 20,13 & 22,04 & 7,80 & 5,32 & 2,91 & 12,41 \\
\hline$p(\%)$ & 0,000 & 0,000 & 0,000 & 0,000 & 0,000 & 0,001 & 0,000 \\
\hline \multirow[t]{3}{*}{$\mathrm{D}=\sigma_{p}^{2} / \sigma_{f}^{2}$} & 0,236 & 0,266 & 0,324 & 0,134 & 0,090 & 0,042 & 0,186 \\
\hline & & & - - & $\cdots$ & - & & $\cdots \cdots$ \\
\hline & $A G V I$ & AGVS & GRVI & GRVS & MATCOC & SUR89 & SUR93 \\
\hline - & & $\cdots$ & & & & & \\
\hline \multicolumn{8}{|l|}{ Analyse 1} \\
\hline F & 2,49 & 1,69 & 2,17 & 1,510 & 3,21 & 1,22 & 2,72 \\
\hline$p(\%)$ & 0,490 & 7,184 & 1,536 & 12,408 & 0,036 & 27,181 & 0,286 \\
\hline $\mathrm{D}=\sigma_{p}^{2} / \sigma_{f}^{2}$ & 0,040 & 0,019 & 0,031 & 0,014 & 0,058 & 0,013 & 0,093 \\
\hline \multicolumn{8}{|l|}{ Analyse 2} \\
\hline $\mathrm{F}$ & 3,15 & 3,90 & 8,33 & 10,47 & 41,16 & 30,46 & 35,64 \\
\hline$p(\%)$ & 0,042 & 0,002 & 0,000 & 0,000 & 0,000 & 0,000 & 0,000 \\
\hline $\mathrm{D}=\sigma_{p}^{2} / \sigma_{f}^{2}$ & 0,049 & 0,065 & 0,149 & 0,184 & 0,489 & 0,657 & 0,693 \\
\hline \multicolumn{8}{|l|}{ Analyse 3} \\
\hline $\mathrm{F}$ & 3,71 & 3,05 & 6,75 & 6,40 & 27,31 & 14,21 & 18,78 \\
\hline$p(\%)$ & 0,000 & 0,000 & 0,000 & 0,000 & 0,000 & 0,000 & 0,000 \\
\hline $\mathrm{D}=\sigma_{p}^{2} / \sigma_{f}^{2}$ & 0,058 & 0,045 & 0,116 & 0,109 & 0,374 & 0,439 & 0,513 \\
\hline
\end{tabular}

L'analyse 1 est faite sur les provenances du sud-est : test $F$ à 11 et 422 ddl (11 à 189 pour SUR89 et 93). L'analyse 2 est faite sur 13 provenances de l'aire de distribution (dont 2 du sud-est) : test $F$ a 11 et 513 ddl (12 et 191 pour SUR89 et 93). L'analyse 3 est faite sur la totalité de l'échantillon de provenances : test $F$ à 23 et 1042 ddl (24 et 399 pour SUR89 et 93). $p(\%)$ : probabilité associée au test $F$ (en pourcentage) ; D : $\sigma_{p}^{2 /} \sigma_{f}^{2}$ est le rapport de la composante provenance à la variance totale. 
sucoccus feytaudi et la survie en 1993 avec, néanmoins, l'existence d'un certain effet d'échelle géographique, le facteur provenance étant nettement plus significatif dans le cas de l'analyse effectuée sur l'ensemble de l'aire de l'espèce (analyse 2).

La variabilité à l'échelle régionale (analyse 1) est, à l'inverse, beaucoup plus faible pour tous les caractères et non significative pour les caractères de forme (écart à la verticalité basale et coefficient de forme) ainsi que pour la survie en 1989. L'intégration des 3 provenances des Corbières et de la provenance du Gard à l'échantillon de provenances autochtones accroît la valeur des tests $F$ jusqu'à les rendre significatifs pour l'ensemble des caractères (données non présentées).

Le rapport $\sigma_{p}^{2} / \sigma_{t}^{2}$, que nous notons $\mathrm{D}$ pour la suite, peut être pris comme indice de différenciation des populations : plus sa valeur sera élevée, plus les populations seront différenciées. II est fonction de l'échelle et de la densité d'échantillonnage comme le montrent les résultats obtenus ici. En effet, on remarquera des valeurs de $\mathrm{D}$ croissant depuis le niveau régional (analyse 1) jusqu'à celui de l'aire totale (analyse 2 ), ceci pour la plupart des caractères.

L'analyse 3 , bien que beaucoup plus proche de l'analyse 2 , aboutit à des résultats intermédiaires dus à la surreprésentation de populations géographiquement voisines, ce qui met en évidence un effet de la densité d'échantillonnage sur D. Mais D peut être interprété en termes biologiques : le niveau de différenciation des populations du sud-est est nettement plus faible que celui de l'ensemble de l'aire. Cependant, on notera que les valeurs de $\mathrm{D}$ sont nettement plus élevées, quel que soit le niveau d'échantillonnage, pour la survie (SUR93), les hauteurs ( $\mathrm{H} 88, \mathrm{H} 89$ et $\mathrm{H} 93$ ) et la sensibilité à $M$ feytaudi que pour les autres variables prises en compte. Pour SUR89 et l'écart à la verticalité (EV88), D est très faible au niveau régional. Par souci de concision nous ne détaillerons ci-après que les résultats issus de l'analyse 3. La figure 1 présente les moyennes de provenances, leurs erreurs standard et les résultats du test de comparaison multiple (Newman et Keuls) au seuil de risque de $5 \%$ pour H93, EV88 et MATCOC. Pour ce test, les variables ont été retransformées en leur unité d'origine.

\section{Adaptation}

\section{Survie}

Les taux de survie à 8 et 9 ans étant très voisins, seuls les résultats concernant SUR89 sont présentés. L'analyse de variance montre des différences très significatives pour les observations faites à 9 et 13 ans.

Le classement et la comparaison des fréquences de survie au seuil de risque de $5 \%$ pour SUR89 opposent globalement TAB $(25,70 \%)$ à l'ensemble des autres provenances qui enregistrent $78,07 \%$ (EST) et $100 \%$ de survivants. Ces performances ne sont pas simples à expliquer car les facteurs qui ont pu les déterminer ne sont probablement pas identiques pour toutes les provenances. Les grands froids de l'hiver 1985 sont très vraisemblablement impliqués. En outre, si l'on admet que la sécheresse estivale est quasi permanente dans les conditions de l'expérience, on peut également attribuer une part de la mortalité à cet élément. Aux mortalités dues aux traumatismes et à la crise de transplantation près, ces 2 facteurs pourraient expliquer une grande partie de la variabilité inter-provenances. On observe, pour SUR93, une amplification des différences entre provenances en même temps que se dessine la constitution de groupes un peu plus tranchés mais néanmoins se chevauchant:

- i) un premier groupe constitué de 6 provenances (TAM, LEI, CAZ, NAV, ROC(a), $\left.P O R^{(a)}\right)$ qui expriment une fréquence de 


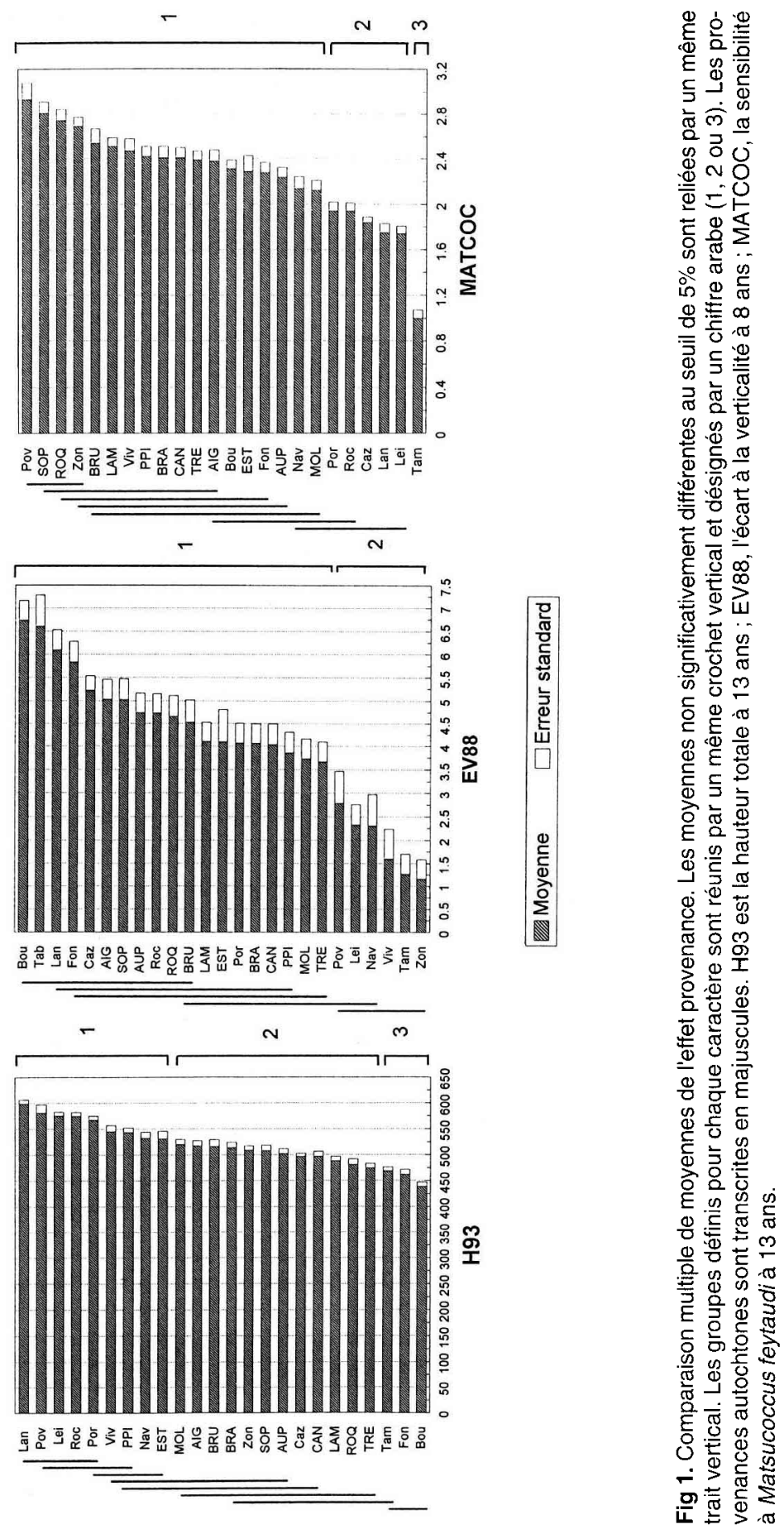


survie supérieure à $80 \%$ (près de $100 \%$ pour TAM) ;

- ii) un second groupe affichant une survie de 52 à $78 \%$ et qui comprend 14 provenances sur un total de 25 (VIV, LAN, BOU(a), ZON, LAM, TRE, FON(a), CAN, PPI, EST, MOL, AUP, POV) ;

- iii) un troisième groupe réunissant des provenances (SOP, AIG, ROQ, BRU) dont le taux de survivants varie de 34 à $48 \%$;

- iv) et, enfin, un quatrième ne comportant qu'une seule provenance (TAB) avec $0 \%$ de survie.

Les changements survenus entre 1989 et 1993 touchent principalement les provenances de la race locale, les provenances de la race corse ainsi que Tabarka. La mortalité entre les 2 dates d'observation est forte : de l'ordre de 30 à $40 \%$ pour les premières, de 20 à $30 \%$ pour les secondes et aénantissement total pour la dernière. Ces résultats coïncident avec l'apparition de $M$ feytaudi, signalé pour la première fois en 1990 par Schvester (communication personnelle) qui faisait état de «symptômes non équivoques d'attaques" par cet insecte. Étant donné le cycle biologique de l'insecte et les symptômes en çjestion, les premières attaques remonteraient à 1989 , soit à l'âge de 9 ans pour les plants.

\section{Sensibilité à Matsucoccus feytaudi}

L'analyse de variance met en évidence un effet provenance global hautement significatif $(F=27,31$ correspondant à une probabilité inférieure au 1:10 000 $)$ ) soulignant une différenciation très élevée des populations naturelles pour la sensibilité à la cochenille. Comme la variance du caractère pour TAM est nulle, l'analyse a été réalisée avec puis sans cette provenance. Le test $F$ dans le deuxième cas était égal à 18,3 , toujours très significatif à un risque calculé du même ordre de grandeur que précédemment. Le test de comparaison des notes moyennes (notons que le classement des provenances sur la base des pourcentages d'individus sains (note $=1$ ) est très semblable à celui fait sur les notes moyennes) au seuil de risque de $5 \%$ révèle approximativement l'existence de 3 groupes de provenances au comportement différent vis-à-vis de l'insecte (fig 1) :

-i) un premier groupe que l'on peut qualifier de très sensible à $M$ feytaudi (note moyenne variant de 2,93 à 2,12 ) se compose des provenances de la race locale des Maures et de l'Estérel, des provenances corses et des provenances NAV (Vieille Castille), BOU et FON (Corbières). Ces dernières sont néanmoins les moins sensibles du groupe;

- ii) un deuxième groupe, constitué de provenances occidentales ou supposées telles (atlantiques et ibériques: POR(a), ROC(a), CAZ, LAN), présentent une certaine résistance à l'insecte (notes de 194 à 1,74), avec un pourcentage d'individus sains non négligeable pour certaines d'entre elles;

- iii) le troisième groupe comporte une provenance marocaine de la race de l'Atlas (TAM) qui présente $100 \%$ d'individus sains.

En termes de pourcentage d'individus sains (note $=1$ ), TAM se classe très nettement en tête avec $100 \%$ suivie par LEI $(36,57 \%)$, CAZ $(25 \%)$, LAN $(24,51 \%)$ et $\operatorname{ROC}^{(a)}(20,37 \%)$; cette dernière, rappelons-le, serait une provenance artificielle d'origine atlantique. Toutes ces races ou provenances colonisent la partie occidentale de l'aire de l'espèce. À l'opposé, SOP, $\mathrm{BRU}, \mathrm{PPI}$, provenances de la race locale, ainsi que VIV (Corse) ne montrent aucune forme de résistance à l'insecte à l'âge de 13 ans ( $0 \%$ de sujets sains) alors que les autres populations de la partie orientale de l'aire affichent un maximum de $11 \%$ (MOL). Remarquons que, pour TAB, il ne subsiste plus qu'un individu complètement dépéri (note = 5). La figure 2 montre l'importance relative des 5 classes de sensibilité à Matsucoccus feytaudi pour les races représentées dans l'expérience. Afin d'éviter la 


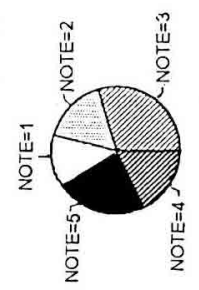

응 옹

过

亏ั

응

क 木

홍

등. 엔

흥 엉

$\Phi$ 침

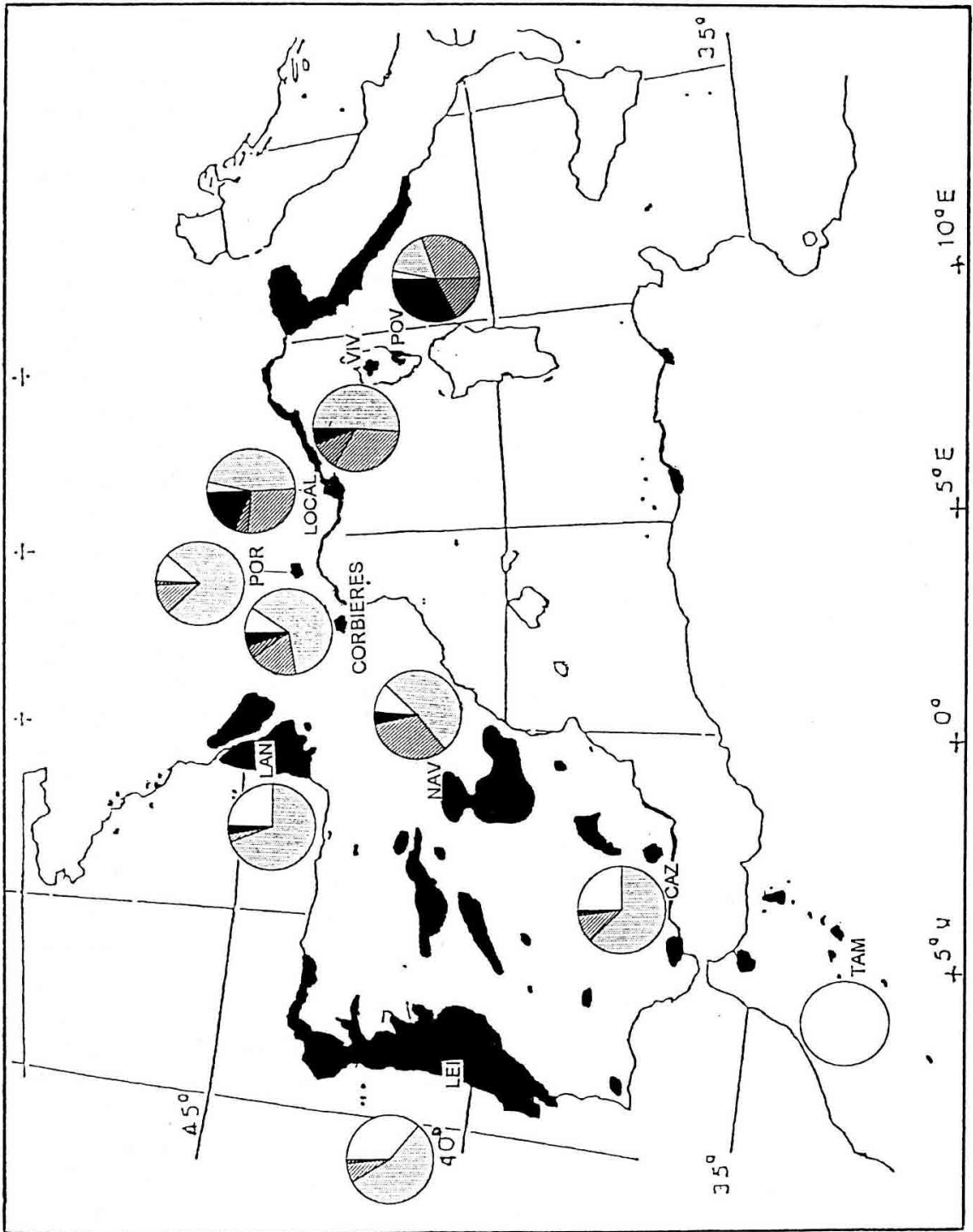

咂

के

또에

흥응

용

을 을

ว

항

要

$\pm$

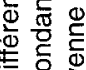

호잉

용 을

능

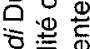

政

क्षे

$\underset{\infty}{\Phi}$

疍 응

O

원

की

$\sum \pi$

. $\frac{1}{0} \frac{1}{9}$

몽

훙

क⿻

\%응

๑ \$

过

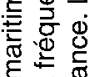

는

高宁崩

긍 $\frac{0}{\overline{0}}$

등 홍

흔 능 홍

홓융흥

$8 \frac{5}{8}$

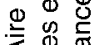

๘

柁密高 
surcharge de la figure, la race du sud-est ainsi que les provenances des Corbières sont illustrées par un diagramme moyen. Nous pouvons faire les constatations suivantes:

- les provenances ne tendent pas vraiment à s'agréger en fonction de leur appartenance aux 3 grands ensembles géographiques basés sur la diversité terpénique. En effet, on trouve réunies dans le même groupe de sensibilité des entités appartenant aux divers ensembles ;

- il semble se dessiner une variabilité en "gradient longitudinal», les provenances occidentales de l'espèce étant nettement plus résistantes à l'insecte que leurs homologues orientales.

La régression multiple descendante de la note moyenne sur le jeu de variables géographiques - latitude, longitude et altitude apporte un argument numérique à cette observation. L'altitude, qui est éliminée dès le premier palier n'explique en rien la variation de la sensibilité à l'insecte (coefficient de corrélation multiple de 0,783 aussi bien pour 3 que pour 2 variables explicatrices). La variable latitude, éliminée au $2^{e}$ palier, fait chuter très modérément ce coefficient de 0,783 à 0,772 qui correspond à la valeur du coefficient de corrélation simple entre la note de sensibilité et la variable longitude $\left(r=0,772^{\star \star \star}\right.$, pour $\left.22 \mathrm{ddl}\right)$. Cette variable explique ainsi la majeure partie de la variation du caractère.

Ce gradient de sensibilité se superpose à la distribution géographique de $M$ feytaudi établie par Riom (1980) et qui montre une présence quasi générale du ravageur dans la partie occidentale et son absence dans la partie la plus orientale de l'aire. Si, de plus, on tient compte des résultats de Schvester et Ughetto (1986), qui montrent la résistance de Cuenca (Espagne) et la sensibilité de Gènes (Ligurie), ainsi que ceux de Harfouche et al (1994b, soumis à publication), qui font état de la sensibilité de Gènes et de Tabarka (Tunisie) sur la base des per- formances de leurs descendances hybrides, on peut avancer, sans risque d'erreur majeur, que ce gradient concerne l'ensemble de l'aire de l'espèce.

II semble, à la lumière de tous ces résultats, que la progression de l'insecte se soit opérée à partir de populations occidentales, plus précisément à partir du Maroc où l'insecte est endémique et probablement d'installation très ancienne (Riom, 1980). Schvester (1981), pour sa part, était arrivé à conclure que le ravageur a été introduit en région méditerranéenne en provenance de la zone atlantique.

\section{Vigueur}

\section{Hauteur totale}

Ce caractère s'avère l'un des plus discriminants entre provenances. On remarque un accroissement de la proportion de variance expliquée par l'effet provenance au cours du temps (voir valeurs de $D$ pour $\mathrm{H} 88$, H89 et $\mathrm{H} 93$ : tableau III), qui traduit une accentuation des différences entre provenances avec l'âge et donc une réduction proportionnelle de la variation intra-provenances. Ce résultat pourrait s'expliquer par le fait que les plants, à l'état juvénile, sont individuellement soumis aux micro-variations du milieu, probablement très prononcées, alors que, plus âgés, ils sont plutôt confrontés à des variations environnementales moyennes qui s'exercent à l'échelle de la plantation.

Les classements pour les 3 mesures de hauteur sont analogues, les provenances montrant une stabilité relative à l'exception de Zonza qui régresse du $9^{e}$ rang en 19881989 au $1^{\circ}$ rang en 1993 et de Navaleno qui remonte, au contraire, de la $15^{\mathrm{e}}$ place en 1988-1989 à la $8^{\mathrm{e}}$ en 1993 . On peut, globalement, distinguer 3 agrégats de provenances (fig 1) évoquant plus ou moins bien les groupes géographiques définis sur la base du profil terpénique : 
- i) un premier groupe de tête rassemble les provenances atlantiques : Landes, Leiria, Lesignan2 (ROC(a)), Portes (POR(a)) et Navaleno, les provenances corses : PortoVecchio et Vivario, et enfin Plan Pinet, provenance des Alpes-Maritimes ;

- ii) un groupe moyen est formé exclusivement de provenances périméditerranéennes ;

- iii) un dernier groupe est constitué par 2 provenances nord-africaines: Tamjout et Tabarka (cette dernière avait totalement disparue en 1993), auxquelles viennent s'ajouter Lesignan1 (BOU(a) et Fonfroide (FON(a)), supposées appartenir au groupe atlantique en référence à leur profil terpénique (Baradat et Marpeau-Bezard, 1988).

\section{Diamètres}

D1.3 et D0.5 sont assez discriminants $(F=$ 7,8 et 5,3 respectivement) mais ils ne déterminent aucune structuration des populations en entités géographiques distinctes. Ces 2 caractères sont probablement affectés par l'existence de trouées dans le dispositif. Les classements présentent beaucoup d'analogies pour les 2 caractères. Pour l'un comme pour l'autre, les provenances CAZ, TAM, POV et NAV sont celles dont la croissance radiale est la plus forte. À l'inverse, BOU(a), TRE, AUP, LAM et ROQ ont des diamètres nettement plus faibles.

\section{Forme}

\section{Écart à la verticalité basale (EV88)}

Le test $F$ pour ce caractère révèle une différenciation entre races et provenances hautement significative. Le test de Newman et Keuls permet de distinguer 2 groupes autour d'une moyenne générale de 4,09 degrés d'écart à la verticalité (fig 1) :

- i) Un groupe formé des provenances corses (ZON, VIV et POV), marocaine (TAM), castillane (NAV) et portugaise (LEI), qui s'avèrent les meilleures pour la rectitude du tronc. Parmi ces provenances, ZON, TAM et VIV enregistrent des performances inférieures à 1,6 degrés.

- ii) Un groupe constitué de toutes les autres provenances dont les performances vont de 6,73 pour la plus mauvaise $(B O U(a))$ à 3,7 pour la moins mauvaise (TRE).

\section{Coefficient de forme $(f)$}

Pour ce caractère, le test $F$ bien que significatif $(2,9)$ n'est pas très loin du $F$ limite $\left(F_{l}=1,87\right)$. Cependant, les valeurs de $F$ s'échelonnent de 0,57 pour $B O U^{(a)}$ et $Z O N$ à 0,47 pour $C A Z$ avec une moyenne générale de 0,52 .

\section{Angles d'insertion des branches}

Nous ne considérerons que l'angle d'insertion des verticilles inférieurs (AGVI). Ce caractère est peu discriminant même si le test $F$ se révèle significatif, ce qui est en accord avec les résultats obtenus pour d'autres espèces comme le pin laricio (Charon, communication personnelle).

Le classement et les différences significatives au seuil de risque global de $5 \%$ montrent que les provenances se distribuent autour d'une note moyenne de 1,70 pour une étendue de 0,67 soit environ $39 \%$ de la moyenne. Bien qu'il n'y ait pas de tendance forte à l'agrégation en groupes, on peut quand même distinguer quelques provenances et races aux comportements extrêmes. EST, BOU(a), et TRE (provenance locale) développent des branches assez horizontales (notes moyennes respectives de $1,37,1,38$ et 1,47 qui correspondent à des valeurs angulaires situées autour de $15^{\circ}$ ). À l'opposé, on trouve SOP et 2 provenances corses (POV et ZON) avec des notes moyennes légèrement supérieures à 2, soit une valeur angulaire moyenne de $24^{\circ} 5$ (classe de 15 à $30^{\circ}$ ). La performance des 2 provenances corses peut surprendre étant donné leur réputation de 
morphotypes de qualité aussi bien pour la rectitude du fût que pour l'horizontalité des branches (Illy, 1966). II faut croire, cependant, à une variabilité au sein même de cette race, la troisième provenance (VIV) étant bien distincte des 2 premières.

\section{Grosseur des branches}

Aussi bien pour GRVI que pour GRVS, I'analyse met en évidence une variabilité importante entre provenances. Le classement des provenances et le test de comparaison de leurs moyennes au seuil de risque de $5 \%$ pour les 2 caractères sont très comparables.

L'étendue de la distribution des moyennes est de 0,870 pour une moyenne générale de 1,991 , soit une étendue relative d'environ $40 \%$, ce qui traduit un regroupement relativement fort autour de cette valeur centrale. II ne se dégage pas, en effet, de tendance à la formation de classes très contrastées. On peut néanmoins relever que LEI, LAN ainsi que 2 provenances corses (VIB et ZON) sont parmi les provenances aux branches les plus fines. À l'inverse, la troisième provenance corse (POV) et les provenances $\mathrm{CAZ}, \mathrm{AIG}, \mathrm{BOU}(\mathrm{a})$ et TAM sont celles qui exhibent les branches les plus grosses. Les provenances de la race locale montrent des performances intermédiaires. On peut faire la même remarque que pour $A G V I$ concernant le comportement des provenances corses : il existe des différences significatives entre elles puisque leurs rangs vont du fer (POV) aux $20^{e}$ et $22^{e}$ (ZON et VIV). Ces résultats viennent encore contredire le fait, en général admis, d'une supériorité de ces provenances pour la qualité de leur branchaison.

\section{Liaisons entre caractères au niveau provenance}

Les corrélations totales (tableau IV) ont été calculées sur la base des moyennes de pro- venances issues des données ajustées à l'effet bloc. Pour 22 ddl, les valeurs limites de $r$ sont respectivement égales à $0,404(\alpha=$ $0,05), 0,515(\alpha=0,01)$ et $0,629(\alpha=0,001)$.

On relèvera, tout d'abord, les très fortes corrélations entre les 3 mesures de hauteur qui s'expliquent, en partie, par les autocorrélations mais qui traduisent sans doute également une relative stabilité du pool génique dans l'intervalle de temps considéré. Bien que positives, les corrélations entre hauteurs et diamètres ne sont pas significatives. On soulignera, en outre, la liaison inverse mais significative au seuil $\alpha=0,01$ entre la hauteur $(\mathrm{H} 93)$ et la grosseur des branches aux verticilles inférieurs de l'arbre. On n'observe pas, en revanche, de liaison forte entre la hauteur et l'angle d'insertion des branches bien que celle-ci soit assez voisine du seuil de signification $(0,39$ vs 0,40$)$. L'écart à la verticalité ne semble lié de façon notable à aucun des autres caractères. Mention peut être faite d'une corrélation négative, néanmoins non significative, avec les 2 mesures de diamètres.

L'étude des corrélations entre la sensibilité à Matsucoccus feytaudi, d'une part, et les caractères de vigueur ou de forme, d'autre part, ne met en évidence aucune liaison statistiquement significative. On remarque, en revanche, la liaison hautement significative avec la mortalité sur la période 1989-1993 ( $r=0,74)$ d'une part et SUR93 $(r=-0,81)$ d'autre part, ce qui vient confirmer l'hypothèse émise plus haut selon laquelle l'action du ravageur se serait manifestée à partir de 1989-1990. La sensibilité des jeunes arbres à l'insecte apparaît d'ailleurs vers l'âge de 10 ans (Schvester et Ughetto, 1986).

\section{Discrimination multicaractère des races et provenances (tableau $\mathrm{V}$ et fig 3)}

Les 3 premiers axes expliquent $82 \%$ de l'inertie totale du nuage de points et leurs 


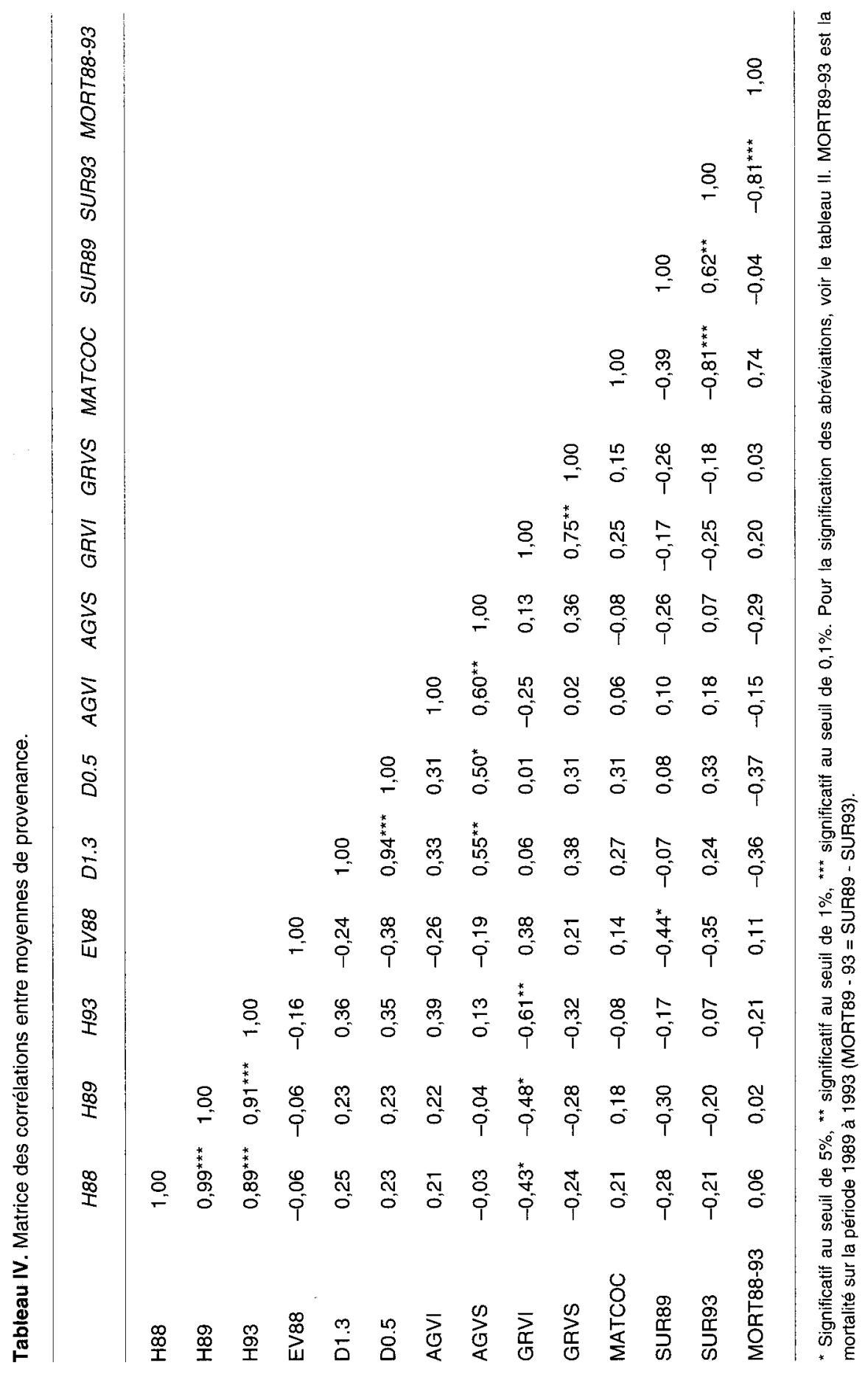




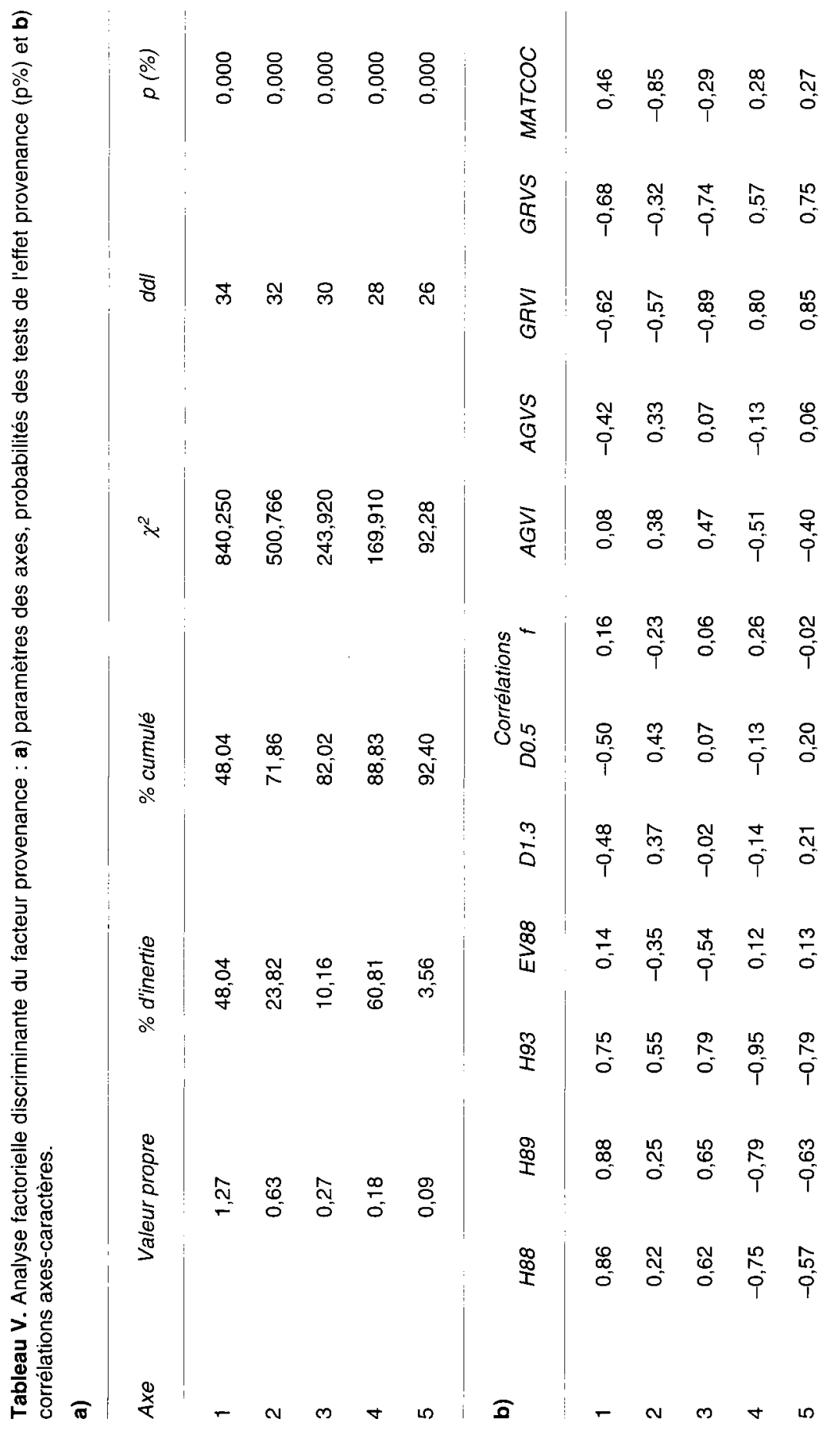




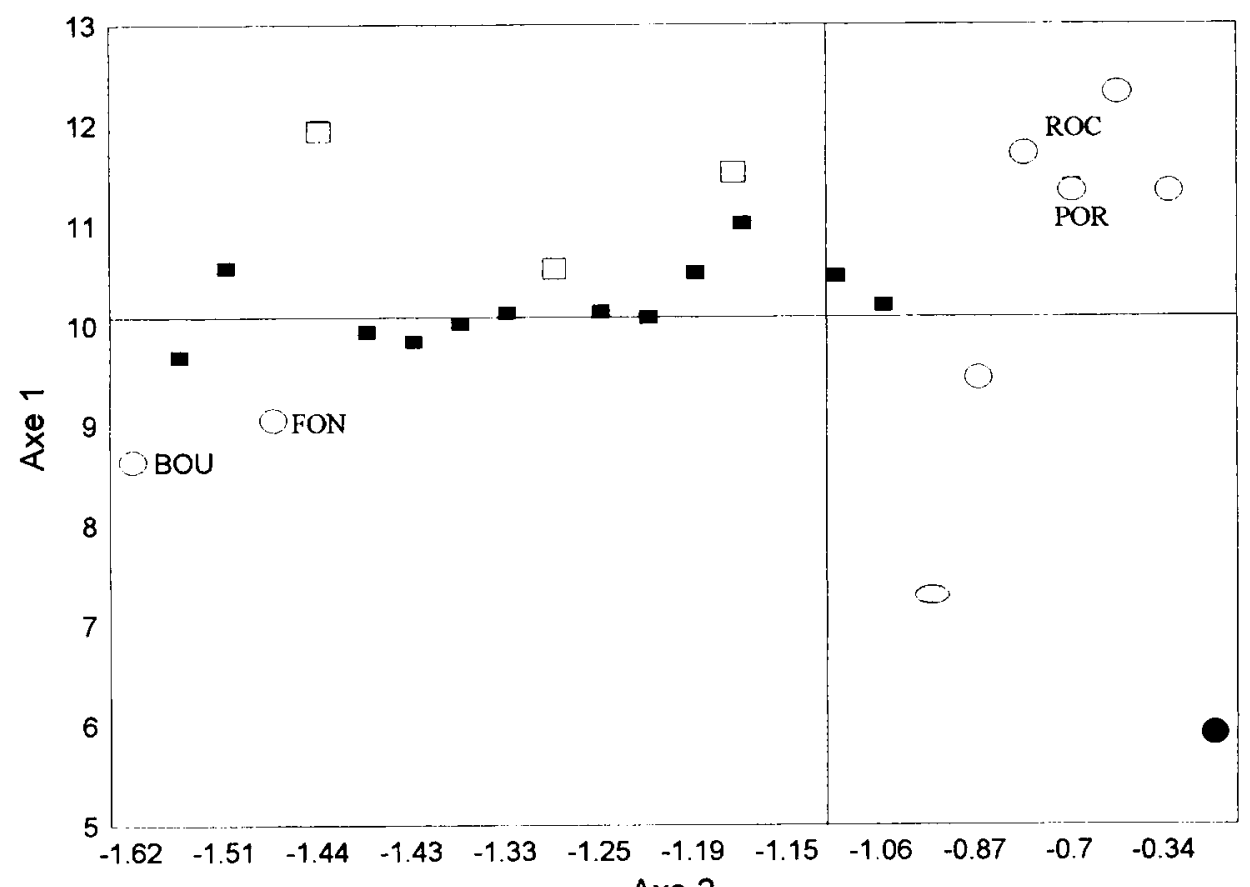

Axe 2

Fig 3. Projection des populations sur les 2 premiers axes de l'analyse factorielle discriminante. O Atlantique ; Périméditerranéen du sud-est de la France ; $\square$ Périméditerranéen corse ; Périméditerranéen andalou ; Maghrébin.

tests sont tous hautement significatifs à une probabilité de l'ordre du $1 / 10000^{\mathrm{e}}$. II faut, cependant, bien observer que les hauteurs et les grosseurs des branches sont des caractères qui présentent des poids importants sur la plupart des axes et qui apportent ainsi une information plus ou moins redondante. Le premier axe discrimine très fortement les provenances et absorbe à lui seul plus de $48 \%$ de l'inertie totale. Il est lié fortement et positivement à $\mathrm{H} 88, \mathrm{H} 89$ et $\mathrm{H} 93$ et, dans une moindre proportion et négativement, à GRVI et GRVS. Les provenances LAN et TAM se situent aux extrémités de cet axe. Le second axe, qui représente environ $24 \%$ de l'inertie, est associé presque exclusivement à la sensibilité à $M$ feytaudi. TAM y occupe une place particulière correspondant à une sensibilité mini- male. Le troisième axe explique $10 \%$ de l'inertie totale et se trouve corrélé négativement à GRVI, GRVS et EV88 et positivement à H93. Le plan 1-2 (fig 3) résume l'essentiel de l'information et permet de mettre en évidence 3 agrégats de provenances qui n'évoquent qu'imparfaitement les 3 grands groupes géographiques. Au sein du groupe périméditerranéen, les provenances de la race corse se distinguent plus ou moins de celles de la race du sud-est. De plus, CAZ, race andalouse occupe une position intermédiaire entre les groupes périméditerranéen et maghrébin, ce dernier n'étant représenté, il est vrai, que par la seule provenance TAM. II faut savoir que ces 2 provenances sont des écotypes de montagne sèche poussant sur le même type de substrat dolomitique. Notons enfin que, si 
$\mathrm{POR}^{(\mathrm{a})}$ et ROC(a) s'intègrent bien au groupe atlantique, BOU(a) et FON(a) en sont, en revanche, très éloignées.

\section{DISCUSSION}

\section{Niveau de différenciation et organisation de la variabilité géographique}

Cette étude met en évidence une importante différenciation des populations naturelles pour les caractères de vigueur et la sensibilité à la cochenille du pin maritime tant au sein de la race locale du sud-est qu'à l'échelle de l'aire de l'espèce. Le modèle de variation en mosaïque du pin maritime semble ainsi se réitérer à diverses échelles pour ces caractères avec, cependant, une variabilité plus importante entre populations de régions différentes qu'entre populations de la même région géographique. En revanche, les populations autochtones sont très peu différenciées pour les critères de forme. Pour les caractères de vigueur et de forme, la configuration de la variabilité géographique que nous avons observée dans le sud-est de la France est très comparable à celle constatée dans le sud-ouest (Guyon, 1980 ; Kremer et Roussel, 1986), au Maroc (Destremau et al, 1976), en Espagne (Gil, 1993), en Australie (Hopkins, 1960) et en Nouvelle-Zélande (Sweet et Thulin, 1962). Les populations atlantiques s'avèrent en effet les plus vigoureuses, les populations corses et marocaines les plus droites. La structuration de la variabilité pour la vigueur correspond par ailleurs à celle qui a été mise en évidence à l'aide des marqueurs terpéniques (Baradat et Marpeau-Bezard, 1988), isoenzymatiques (Petit, 1988) et protéiques (Bahrman et al, 1992).

La supériorité générale des races atlantiques pour la vigueur peut s'expliquer par la taille plus grande des populations qui les constituent, ce qui est de nature à favoriser un brassage des pools géniques et à maintenir une base génétique plus large (probabilité d'hétérozygotie plus grande). Elle peut également résulter de l'action de la sélection naturelle qui a pu favoriser les meilleurs compétiteurs, car, sous les climats atlantiques, il n'existe pratiquement pas de facteurs limitants. Dans les autres parties de l'aire, il s'agit, très fréquemment, d'isolats de faible dimension où des phénomènes de dérive génétique et de consanguinité ont pu modeler les peuplements.

\section{Variabilité géographique clinale de la sensibilité à M feytaudi}

Nos résultats sur des plants âgés de 13 ans sont en accord avec ceux de Schvester et Ughetto (1986) qui avaient également mis en évidence d'importantes différences entre provenances sur des arbres de 20 ans dans un test de provenances implanté dans les Maures (Lambert). Cependant, Riom (1980), sur la base d'observations effectuées dans les Landes et dans les Maures, avait noté que le comportement des diverses provenances vis-à-vis de la cochenille ne semblait pas fondamentalement différent.

La structuration de la variabilité géographique vis-à-vis de la sensibilité à $M$ feytaudi ne répond pas au modèle observé pour les marqueurs biochimiques, moléculaires ou morphologiques. Notamment, elle ne se superpose en aucune façon à la structuration en groupes géographiques mis en évidence sur la base des marqueurs terpéniques. En effet, il semble exister un cline longitudinal ouest-est pour la sensibilité du pin maritime à $M$ feytaudi : les populations occidentales seraient moins sensibles que les populations orientales. Cela nous permet d'émettre quelques hypothèses sur son évolution géographique et de mieux comprendre l'induction d'une forme de résistance chez les populations de pin maritime. 
La mise en évidence de ce gradient de sensibilité ouest-est, qui reste à confirmer par un échantillonnage de provenances plus large et plus représentatif de l'aire de l'espèce, est un argument en faveur d'une installation très ancienne du ravageur dans la partie ouest de l'aire (Riom, 1980) et suggère l'apparition progressive d'une résistance des populations au ravageur par la sélection en faveur des porteurs de gènes de résistance.

Dans les populations de faible taille, comme c'est le cas pour Tamjout, cette pression probablement conjuguée à une dérive aléatoire aurait abouti plus ou moins rapidement à une fixation totale de ces gènes (dans l'hypothèse d'un contrôle polygénique du caractère). Avec la progression de l'insecte vers l'est, le processus se serait reproduit dans les peuplements nouvellement infestés, la fréquence des génotypes résistants augmentant au fur et à mesure de son action. Dans les Maures, où l'introduction du ravageur est assez récente (Schvester, 1981), la sensibilité des populations de pin est grande mais il semble s'y trouver des individus réfractaires à très faible fréquence (Fady, communication personnelle et observation personnelle).

La résistance apparente de ces sujets pourrait être d'origine génétique puisque tous leurs voisins sont fortement attaqués ou dépérissants. Sans intervention extérieure, ces quelques individus seront probablement à l'origine d'une recolonisation du massif par l'espèce. L'augmentation de la fréquence de ces génotypes résistants dans les peuplements constituerait, comme ceux discutés par Schvester (1981), un facteur de rééquilibrage permanent susceptible de contribuer à un freinage des pullulations de l'insecte. L'hypothèse du caractère héréditaire de la résistance de ces arbres pourrait être vérifiée par des tests clonaux et de descendances.

Nous ne disposons pas à l'heure actuelle de moyens sûrs pour résoudre la question d'un contrôle simple ou complexe du caractère de résistance vu la nature du matériel végétal mais certains éléments nous permettent de faire l'hypothèse d'un caractère composite, donc polygénique :

- premièrement, la variation du caractère semble se définir sur une échelle continue. Des degrés de sensibilité sont, en effet, observés sur l'échelle à 5 notes que nous avons retenue. Schvester et Ughetto (1986), pour leur part, certainement dans un souci de mieux rendre compte de la variation observée, avaient adopté une échelle à 7 notes.

- deuxièmement, d'après ces auteurs, la sensibilité des provenances serait en relation avec plusieurs caractères anatomiques (structure des écorces), physiologiques et/ou biochimiques (rejet des tissus lésés par les piqûres chez Tamjout ; réactions sous-épidermiques chez Cuenca, autre provenance résistante originaire d'Espagne du sud-est).

\section{Intérêt des populations autochtones}

Un résultat important de l'étude est le comportement tout juste moyen des populations autochtones qui sont fréquemment surclassées par des races "exotiques» même pour des caractères définissant la valeur adaptative des génotypes. Ce résultat pose la question de l'intérêt de la race locale pour le développement sylvicole de la région méditerranéenne française et de son implication dans le cadre d'un programme régionalisé d'amélioration génétique de l'espèce. Il est, à notre avis, évident qu'elle ne pourra y jouer qu'un rôle très marginal, du moins à court et moyen termes. L'action à court terme à entreprendre en faveur de ces écotypes méditerranéens ne peut être qu'une mesure de conservation ex situ ou in situ pour les rares individus apparemment résistants à $M$ feytaudi dans la mesure où cette résistance se révélerait d'ordre génétique. 


\section{Intérêt de la filière hybrides interraciaux}

La différenciation importante et la complémentarité entre les races et provenances de pin maritime pour les caractères les plus importants mises en évidence par l'étude laissent espérer une efficacité d'une hybridation interraciale chez cette espèce. Cette filière nous semble préférable à un simple transfert de population au moins pour 2 raisons :

- i) Aucune des provenances testées ne nous parait répondre à l'idéotype que l'on peut souhaiter pour la zone du sud-est. Si Tamjout semble convenir pour ce qui est de la résistance à $M$ feytaudi, elle ne saurait en revanche faire l'unanimité pour les caractères de vigueur et de forme que la sylviculture est en droit d'attendre.

- ii) Les hybrides, par leur plus grande stabilité phénotypique vis-à-vis des variations environnementales, offrent une plus grande garantie contre les accidents naturels et les changements imprévisibles qu'ils soient d'origine climatique ou biologique.

II reste à dire que les résultats obtenus sont à confirmer en raison des limites inhérentes à l'échantillon de provenances étudiées (faible représentation de certaines parties de l'aire) ou au caractère mono-site de l'expérience, qui est loin de couvrir la diversité des situations écologiques du sudest de la France. Ils doivent, en outre, s'avérer répétables à des âges plus avancés, en particulier au-delà de la phase de régulation des infestations ; c'est-à-dire vers 15-16 ans selon Schvester (1981).

\section{REMERCIEMENTS}

Nos remerciements vont à B Fady, directeur du domaine expérimental du Ruscas et à toute son équipe pour leur formidable accueil. Mention spéciale à l'équipe technique pour son application dans les mesures.

\section{RÉFÉRENCES}

Bahrman N, Baradat P, Petit R (1992) Structuration de la variabilité génétique du pin maritime dans l'ensemble de son aire naturelle. Hypothèses explicatrices. In : Complexes d'espèces, flux de genes et ressources génétiques, Colloque International en hommage à Jean Pernès, BRG/MRT Paris, 351368

Baradat P, Marpeau-Bezard A (1988) Le pin maritime Pinus pinaster Ait. Biologie et génétique des terpènes pour la connaissance et l'amélioration de l'espèce. Thèse doct État, univ Bordeaux I

Bouvarel P (1960) Note sur la résistance au froid de quelques provenances de pin maritime. Rev For Fr $12,495-508$

Dagnelie P (1973) Théorie et méthodes statistiques. Presses agronomiques de Gembloux, Gembloux

Destremau DX, Joly H, Tahri T (1976) Contribution à la connaissance des provenances de Pinus pinaster. Ann Rech For Maroc 16, 101-153

Gil L (1993) Genetic improvement of Pinus pinaster. variability in mediterranean Spain. International course of ERASMUS: Development of fast growing forest species and their effect on the environnment. Bordeaux (France) 21 June-2 July 1993

Guyon JP (1980) Variabilité géographique et écophysiologique du pin maritime. Mémoire de fin d'études ENITEF

Hopkins ER (1960) Variation in the growth rate and quality of Pinus pinaster Aiton in Western Australia. West Austr For Dep Bull 67, $34 \mathrm{p}$

Illy G (1966) Recherches sur l'amélioration génétique du pin maritime (Pinus pinaster Ait). Ann Sci For 23 , 757-948

Kremer A, Roussel G (1986) Décomposition de la croissance en hauteur du pin maritime (Pinus pinaster Ait). Variabilité géographique des composantes morphogénétiques et phénologiques. Ann Sci For 43, 15-34

Lellouch J, Lazar P (1974) Méthodes statistiques en expérimentation biologique. Flammarion, Médecine Science, Paris

Pearce SC (1965) Introduction à la statistique en biologie. INRA, Paris (traduction de JP Doazan, 1978)

Petit $R$ (1988) Ressources génétiques du pin maritime: apport des marqueurs enzymatiques. DEA, université Paris-Sud-Orsay

Resch T (1974) Essai de distinction anatomique des races majeures de Pinus pinaster. Ann Rech For Maroc 14, 91-102

Riom J (1980) Biologie et écologie des populations de la cochenille du pin maritime Matsucoccus feytaudi Ducasse 1942 (Coccoïdea margarodidae). Thèse doct État, univ Bordeaux I 
Schvester D (1981) Pin maritime et Matsucoccus feytaudi Duc. État actuel de la question. For Med III $\mathrm{n}^{\circ} 2$

Schvester D, Ughetto F (1986) Différences de sensibilité à Matsucoccus feytaudi Duc (Homoptera: Margarodidae) selon les provenances de pin maritime (Pinus pinaster Ait). Ann Sci For 43, 459-474

Snedecor GW, Cochran WG (1957) Méthodes statistiques. Association de coordination technique agricole (traduction de $\mathrm{H}$ Boelle et $\mathrm{E}$ Camhaji) Paris

Sweet GB, Thulin IJ (1962) Provenances of Pinus pinaster Ait. A 5 year progress report on a trial at Woodhill, New Zealand. NZ J For 8, 570-586

Vergeron $P(1970)$ Variabilité entre populations locales de Pinus pinaster Sol. Le problème du pin mésogéen. Document interne INRA, Bordeaux 